\title{
Objectivity in the Study of Intelligence: The Cornerstone of New Methods and Discoveries
}

\author{
Lori Marino
}

Emory University, Atlanta, Georgia 30322

\section{Introduction}

Science requires objectivity - the ability to see the relationships among things in the natural world free of assumptions and biases. To achieve this goal one has to use objective methods of observation and testing but also maintain a philosophy of objectivity when deciding upon which research questions to pursue and which conclusions to draw from one's findings. The field of bioastronomy relies upon objectivity on both a methodological level and, perhaps to the greatest extent among all sciences, on a conceptual level. Bioastronomy is the multidisciplinary and interdisciplinary science of investigating, understanding, and explaining the origin, nature, prevalence, and distribution of life in the universe. As a scientific field of inquiry bioastronomy relies upon methodological objectivity. Furthermore, bioastronomy demands conceptual objectivity because its content domain is, by nature, largely unknown and subject to more degrees of freedom than perhaps any other discipline. Therefore, objectivity, through and through, is the cornerstone of bioastronomy.

The domain of bioastronomy is extremely broad. With the exception of the search for extraterrestrial intelligence, the vast majority of bioastronomy research efforts focus upon very fundamental stages of life in the universe such as organic chemistry, habitable zones, the origin of life, and the evolution of early life on Earth. A smaller proportion of bioastronomy research is devoted to other important domains on the continuum of life such as the study of the evolution of behavior and intelligence, neurosciences, the evolution of complexity, sociality, and technology. One of the reasons why the study of intelligence and complex behavioral processes has played a lesser role in bioastronomy than the study of more basic processes is that intelligence and more complex phenomena are more susceptible, as concepts, to being misunderstood and therefore are more vulnerable to bias. For many people, the study of intelligence and complex processes amounts to confining our efforts to our own species. The problem is that, while intelligence and complex (even technological) behavior are not unique to our own species, they are often viewed as such. This is because of deep and pervasive biases in our perception of humans and other animals. The thesis of this plenary address is that these biases and assumptions limit the objectivity of the study of intelligence and complex behavior within the context of bioastronomy. Three closely related biases or assumptions about intelligence and the nature of the human species are discussed: Scala Naturae thinking, the anthropic principle, and the teleological assumption.

\section{Scala Naturae or Great Chain of Being}

The Scale of Nature (Scala Naturae), also known as the Great Chain of Being, is an idea that is first attributed in writing to Aristotle. The Scala Naturae describes the view that the universe is hierarchically arranged into fixed levels of increasing perfection and authority. The "ladder of progress" denoted by this 
view starts with inanimate objects on the bottom and moves progressively up through plants to "lower" animals, "higher" animals, humans, angels, and a deity. According to this view, humans are separate from and elevated above other animals. Furthermore, humans occupy a special position on the scale straddling the supernatural world as part spiritual being and the physical world as part animal. The propensity for humans to adhere to Scala Naturae thinking is elegantly pointed out in the following quote by de Montaigne (1998): "Presumption is our natural and original disease" This by the same vanity of imagination that [man] equals himself to God, attributes to himself divine qualities, and withdraws and separates himself from the crowd of other creatures".

The Scala Naturae represents an artificial conception of nature inconsistent with the scientific evidence about how organisms are naturally related. The position of human beings above the other animals is entirely a contrivance owing to the fact that human beings devised the scale in the first place. It is logical to assume that if another species, say aardvarks, were in charge of creating a Great Chain of Being that they would position themselves at the top according to what they perceived to be well-justified criteria. Humans would be relegated to, if we were fortunate, the level of "higher" animals. This is not a facetious point. Our position at the top of any kind of progressive scheme is no more scientifically valid than placing aardvarks or any other species there.

At this point one might suspect that I am setting up a strawman argument for the influence of Scala Naturae thinking on modern science. But the Scala Naturae viewpoint, unfortunately, is pervasive, implicitly and explicitly, in scientific writing, popular books, and classroom materials. The language we use betrays the ubiquitous nature of Scala Naturae thinking. For example, the terms "ascent" (despite the fact that we are descendents - not ascendants of our ape ancestors), "higher", "advanced", "highly evolved" are all popular descriptors of our own species in relation to other species. Likewise, terms such as "primitive" and "lower" are still used for organisms that happen to be very different from us. None of these terms are valid reflections of nature and easily evoke the ladder of evolutionary progress represented by the Scala Naturae or Great Chain of Being. The realization that we still rely heavily on a notion about nature that dates from before $300 \mathrm{BC}$ should give us pause.

Within the context of the field of bioastronomy, Scala Naturae thinking manifests itself in a particularly disadvantageous manner. I propose that adherence (often implicitly) to Scala Naturae thinking underlies arguments that intelligence and technology are unique to humans in the universe and therefore not worth pursuing in the context of bioastronomy. This kind of view, which I will term the "N=1 argument" is not a priori invalid or incorrect. We do not, in fact, know whether intelligence of any sort exists off our own planet. However, my objection to the way Scala Naturae thinking influences reasoning about extraterrestrial intelligence is based on the suspicion that it may unduly limit our thinking about extraterrestrial life and thereby inhibit the development of ideas and research within bioastronomy.

\section{The Anthropic Principle}

The Great Chain of Being is a close bedfellow of the Anthropic Principle. The more we come to know about the nature of our universe the more we come to appreciate that so many intricate details of the universe came together to 
provide the kind of natural world that spawned our species. We stand in awe of the seemingly vast improbabilities that underlie our existence. Because of this perception of vast improbabilities we often find ourselves arguing that the universe is somehow tuned or "made for" intelligent life in the form of human beings. This is the basic psychological realization that underlies strong and weak versions of the anthropic principle. The weak form alludes to the obvious fact that, as we are here, conditions must be amenable to our existence. This is an inarguable and trivial point. However, stronger versions of the anthropic principle go further to support the " $\mathrm{N}=1$ " argument by implying that our existence is based upon such vast improbabilities that there could not be another intelligent species somewhere else in the universe. Perhaps more troublesome is the fact that strong versions of the anthropic principle can be used to support arguments that the universe was designed for our existence. Intelligent design and related creationist views preclude, or at the very least severely limit, the ability to conceive of extraterrestrial intelligence highly dissimilar from human. The anthropic principle, therefore, is, at best, a frivolous point and, at worst, a hindrance to objectivity when it comes to issues germane to bioastronomy.

\section{Teleology}

The anthropic principle is closely related to the concept of teleology. Teleology is the study of ends, purposes and goals. The ends of things are viewed as providing the meaning for that which occurs beforehand. Within the context of bioastronomy, the teleological assumption would argue that there is a purposeful progression in cosmic and biological evolution towards better or more advanced organisms. In this view, human beings are the end goal. Teleological assumptions are also expressed in support of intelligent design or creationist views of the universe, both of which are antithetical to objective scientific inquiry.

One of the particular ways in which the teleological assumption is manifest is in our views of human and nonhuman intelligence. The longstanding perception that evolution proceeds from organisms with less complex nervous systems to organisms with more complex brains and greater intelligence is apparently consistent with a broad and rather superficial reading of the evolutionary record across time. It is certainly the case that early in the evolution of life on Earth there were exclusively single-celled organisms without brains and that, just recently, in evolutionary terms, the most highly encephalized organisms on the planet, mammals, emerged. The average brain size for all life on Earth has indeed increased over time. But this is a trivial point because evolution occurs through modification of existing structures and it simply takes time for large and complex structures, like brains, to become an evolutionary option. The deeper and more important question is whether there is a reason to think there is a drive in evolution towards greater intelligence. Even if we were to find evidence for direct selection for increased brain size in several ancestor-descendent lineages we would not be justified in attaching value-laden descriptions to those trends such that increasing encephalization would connote "better" or "more advanced" organisms. Teleological notions implicitly embody these value judgements.

Not unexpectedly, measures of intelligence that we tend to favor are those that place us at an advantage in relation to other species. Humans have the highest level of encephalization on the planet and the most sophisticated technology. We tend to view our specific neuroanatomical attributes as evidence 
for "advancement" while the distinctive features of other species are often considered "primitive". For instance, it is not uncommon for the visual sensory system to be depicted as advanced and the olfactory sensory system as primitive in neuroanatomy textbooks and papers. This is not merely coincidental with the fact that we, as primates, have a sophisticated visual system but a relatively diminished capacity for olfactory processing. The reasoning behind most of these notions about intelligence is circular and self-serving. For every human characteristic that we consider a criterion for the advanced state of our brains we could produce a list of features of the brains of other species that either exceed these criteria or defy the rationale behind choosing these features as indicative of the leading edge of intelligence. For example, dolphins can process sound information ten times faster than humans can, and they possess a perceptual system, based on echolocation, that is absent in us altogether. The neocortex in the dolphin brain is more highly convoluted and has more surface area than the human neocortex. We should acknowledge that these kinds of perceptual-cognitive and neuroanatomical differences make impossible for us to make definitive statements about any species being more advanced than another. In the context of thinking about extraterrestrial life forms, we can't afford to view the human brain in other than an entirely objective comparative light.

All of the biases in thinking (the Scala Naturae, the anthropic principle and the teleological assumption) have important implications for how we think about our place in the universe. These assumptions affect our thinking about extraterrestrial life and intelligence and therefore, the extent and kind of science we do. Although it may be flattering to separate ourselves from the rest of biological evolution and to see our own existence as the pinnacle of some great hierarchy, the fact of the matter is stated straightforwardly by Flanagan (2002): "Humans don't possess some animal parts or instincts. We are animals. A complex and unusual animal, but at the end of the day, another animal".

All of the major paradigm shifts in our understanding of the world have, in one way or another, reassigned our views about ourselves to a more objective stance. This is going to happen within bioastronomy in various domains - and in the most dramatic way if we find life somewhere else. One of the domains in which this is happening already is through the ever-growing realization that naturalistic Darwinian processes are the bases for phenomena such as intelligence and complex behaviors. A more objective kind of scientific revolution is occurring in the study of these processes. This has resulted in the development of more rigorous, quantifiable, and objective investigative methodologies and knowledge in the domains of intelligence, brain evolution, behavior, and other complex processes. Once we get beyond the biases in a real fundamental sense the study of complex behavior and intelligence will form a much larger part of the science of bioastronomy.

\section{References}

Flanagan, O. 2002, The Problem of the Soul: Two Visions of Mind and How to Reconcile Them, (New York: Ed. Basic Books), 3

de Montaigne, M. 1998, Essais de Michel de Montaigne Présentation, Etablissement du texte, apparat critique et notes par André Tournon", (Paris: Imprimerie Nationale) 